\title{
Anharmonic corrections to the electrostatic energy of a Coulomb crystal
}

\author{
A.I. Chugunov*, D.A. Baiko \\ Theoretical Astrophysics Department, A.F. Ioffe Physical-Technical Institute, 194021 St.-Petersburg, \\ Russian Federation
}

Available online 25 January 2005

\begin{abstract}
Model calculations of the electrostatic energy of body-centered-cubic and face-centeredcubic Coulomb crystals are presented. The electrostatic energy is calculated using a pair distribution function of ions in the harmonic approximation. This distribution function is shown to be in a good agreement with the function derived by the Monte Carlo method in the high-temperature classical limit ( $T \gg \hbar \omega_{\mathrm{p}}, \omega_{\mathrm{p}}$ being the ion plasma frequency). It is also in a good agreement with the distribution function obtained by the path integral Monte Carlo method in the low-temperature quantum limit $\left(T \ll \hbar \omega_{\mathrm{p}}\right)$. First- and second-order anharmonic corrections to the electrostatic energy are analyzed in a wide temperature range, from the hightemperature classical regime to the low-temperature quantum regime. For typical conditions in the envelopes of neutron stars and the cores of white dwarfs the anharmonic corrections to the electrostatic energy do not exceed a few percent of the electrostatic energy of harmonic lattice.

(C) 2005 Elsevier B.V. All rights reserved.
\end{abstract}

PACS: 52.27.Lw; 52.27.Gr

Keywords: Coulomb crystals; Harmonic lattice

\footnotetext{
*Corresponding author.

E-mail address: andr.astro@mail.ioffe.ru (A.I. Chugunov). 


\section{Introduction}

The model of a Coulomb crystal of point charges in a uniform neutralizing background of charge of opposite sign is widely used in many branches of physics. In particular, it is often employed in solid-state physics for describing electron-hole plasma (e.g., Ref. [1]) and in plasma physics for describing dusty plasmas and ion plasmas in Penning traps (e.g., Ref. [2]). Moreover, Coulomb crystals of ions with a weakly polarized electron background are formed in the cores of white dwarfs and in the envelopes of neutron stars. Properties of the Coulomb crystals can be analyzed using either purely numerical techniques (simulations), such as classical or path integral Monte Carlo methods, or analytical methods. The latter approach is based on series expansions in powers of displacements of ions from their equilibrium sites, which leads to the harmonic lattice (HL) approximation and phonon formalism (e.g., Ref. [3]). The HL approximation allows one to obtain many useful and sufficiently accurate results, the most obvious example being the energy of ion vibrations in a very broad range of temperatures. Hence, one needs to know the accuracy of the HL approximation.

The answer to this question can be given by calculating the anharmonic corrections to the energy. The exact first-order anharmonic correction to the electrostatic energy of Coulomb crystals with body-centered-cubic (bcc) and facecentered-cubic (fcc) lattices in the classical regime was obtained by Dubin [4]. Later on, Baiko et al. [5] employed an approximate analytical model to calculate the firstand second-order anharmonic corrections to the electrostatic energy of classical Coulomb crystals. The model treated the anharmonic effects in an approximate way. Nonetheless, the agreement between the results of Refs. [4,5] for the first-order anharmonic correction to the electrostatic energy of the classical bcc lattice was very good. Also, the model of Ref. [5] is easily extendable into the quantum regime, provided that ion-exchange effect is unimportant (which is the case of interest in this study). Thus, with this model one can expect to obtain reasonably accurate estimates of anharmonic corrections in the quantum regime, where exact numerical calculations are not available.

It is the aim of the present paper to extend the results of Ref. [5] into the quantum regime and to the case of fcc lattice.

\section{Formalism}

Let us outline the HL model. The electrostatic energy can be written as

$$
\frac{U}{N}=\frac{n}{2} \int \frac{Z^{2} e^{2}}{r}[g(\boldsymbol{r})-1] \mathrm{d} \boldsymbol{r},
$$

where $N$ is the total number of ions in the system, $n$ is the ion number density, $Z e$ is the ion charge, and $g(\boldsymbol{r})$ is the ion pair distribution function. In the HL model $g(\boldsymbol{r})$ 
can be written as (e.g., Refs. [6])

$$
g(\boldsymbol{r})=\sum_{\boldsymbol{R}_{i}}^{\prime} \frac{\sqrt{N\left(\boldsymbol{R}_{i}\right)}}{8 \pi^{3 / 2} n} \mathrm{e}^{-N_{\alpha \beta}\left(\boldsymbol{R}_{i}\right) x_{i \alpha} x_{i \beta} / 4},
$$

where the sum is over direct lattice vectors $\boldsymbol{R}_{i}$ (excluding the central vector $\boldsymbol{R}_{\mathbf{0}}=\mathbf{0}$ as indicated by the prime near the sum) and the vector $\boldsymbol{x}_{i}$ is defined as $\boldsymbol{x}_{i}=\boldsymbol{R}_{i}-\boldsymbol{r}$. Furthermore, $\quad N\left(\boldsymbol{R}_{i}\right)=\operatorname{det}\left\{N_{\alpha \beta}\left(\boldsymbol{R}_{i}\right)\right\}$, with $N_{\alpha \beta}\left(\boldsymbol{R}_{i}\right)=V_{\alpha \beta}^{-1}\left(\boldsymbol{R}_{i}\right)$ and $V_{\alpha \beta}\left(\boldsymbol{R}_{i}\right)=$ $r_{T}^{2} \delta_{\alpha \beta} / 3-v_{\alpha \beta}\left(\boldsymbol{R}_{i}\right)$. The quantity $r_{T}^{2}$ is the mean squared ion displacement in a lattice site, that can be expressed as

$$
r_{T}^{2}=\frac{3 \hbar}{2 M}\left\langle\frac{1}{\omega_{v} \tanh \left(\hbar \omega_{v} / 2 T\right)}\right\rangle_{\mathrm{ph}}
$$

and $v_{\alpha \beta}\left(\boldsymbol{R}_{i}\right)$ is the correlation matrix of displacements of two ions (e.g., Refs. [3,5]),

$$
v_{\alpha \beta}\left(\boldsymbol{R}_{i}\right)=\frac{3 \hbar}{2 M}\left\langle\frac{e_{v \alpha} e_{v \beta}}{\omega_{v} \tanh \left(\hbar \omega_{v} / 2 T\right)} \cos \left(\boldsymbol{q} \cdot \boldsymbol{R}_{i}\right)\right\rangle_{\mathrm{ph}} .
$$

In this case, $v \equiv(\boldsymbol{q}, s)$ labels phonon modes; $\boldsymbol{q}, \boldsymbol{e}_{v}$ and $\omega_{v}$ denote, respectively, the phonon wave vector (in the first Brillouin zone), the polarization vector and the frequency (to be calculated from the standard dynamic-matrix equations). The brackets $\langle\cdots\rangle_{\mathrm{ph}}$ denote averaging over the phonon spectrum, that can be performed numerically using, for instance, the Holas method (e.g., Refs. [7,8]). Finally, $M$ is the ion mass.

The electrostatic energy of a lattice can be divided into three parts: the static (Madelung) energy of the classical ions fixed in their lattice sites, the harmonic energy, and the anharmonic energy. Eq. (1) with $g(r)$ given by Eq. (2) accounts exactly for the static-lattice and harmonic contributions (see below) but approximately describes the anharmonic energy component (as a sum of true Coulomb energies of ions averaged over ion positions with the approximate HL ion pair distribution function e.g. Ref. [5]).

Let us substitute Eq. (2) into (1) and add and subtract the elastic (Bragg) pair correlation function under the integral

$$
\begin{aligned}
g_{1}(\boldsymbol{r}) & =\sum_{\boldsymbol{R}_{i}}^{\prime} \frac{3 \sqrt{3 \pi}}{8 \pi^{2} n r_{T}^{3}} \mathrm{e}^{-3 x_{i}^{2} / 4 r_{T}^{2}} \\
& =1-\frac{3 \sqrt{3 \pi}}{8 \pi^{2} n r_{T}^{3}} \mathrm{e}^{-3 r^{2} / 4 r_{T}^{3}}+\sum_{\boldsymbol{G}_{i}}^{\prime} \mathrm{e}^{i \boldsymbol{r} \cdot \boldsymbol{G}_{i}-r_{T}^{2} G_{i}^{2} / 3} .
\end{aligned}
$$

This function is obtained from $g(\boldsymbol{r})$ by setting $v_{\alpha \beta}\left(\boldsymbol{R}_{i}\right)=0$. In the last term the sum is over inverse lattice vectors $\boldsymbol{G}_{i}$, excluding $\boldsymbol{G}_{\mathbf{0}}=\mathbf{0}$. After introducing $g_{1}(\boldsymbol{r})$, Eq. (1) can be written as

$$
\frac{U}{N}=\frac{n}{2} \int \frac{Z^{2} e^{2}}{r}\left[g_{1}(\boldsymbol{r})-1\right] \mathrm{d} \boldsymbol{r}+\frac{n}{2} \int \frac{Z^{2} e^{2}}{r}\left[g(\boldsymbol{r})-g_{1}(\boldsymbol{r})\right] \mathrm{d} \boldsymbol{r} .
$$


Now we can change the order of summation and integration. Integrating the first term we get

$$
\begin{aligned}
\frac{U_{1}}{N} & =\frac{n}{2} \int \frac{Z^{2} e^{2}}{r}\left[g_{1}(\boldsymbol{r})-1\right] \mathrm{d} \boldsymbol{r}=2 \pi Z^{2} e^{2} n \sum_{i}^{\prime} \frac{1}{G_{i}^{2}} \mathrm{e}^{-r_{T}^{2} G_{i}^{2} / 3}-\sqrt{\frac{3}{4 \pi}} \frac{Z^{2} e^{2}}{r_{T}} \\
& =\frac{Z^{2} e^{2}}{a}\left[\zeta+\frac{r_{T}^{2}}{2 a^{2}}-\sum_{i}^{\prime} \frac{a}{2 R_{i}} \operatorname{erfc}\left(\frac{\sqrt{3} R_{i}}{2 r_{T}}\right)\right],
\end{aligned}
$$

where $\zeta$ is the electrostatic Madelung constant $(\zeta=-0.895929$ for bcc lattice and $=-0.895873$ for $\mathrm{fcc}), \operatorname{erfc}(x)$ is the complementary error function, and $a=$ $(4 \pi n / 3)^{-1 / 3}$ is the ion sphere radius. The last equality is obtained using the expression for the Madelung constant derived by the Ewald method (e.g., Ref. [9])

$$
\zeta=\sum_{i}^{\prime} \frac{a}{2 R_{i}} \operatorname{erfc}\left(\frac{A R_{i}}{a}\right)+\sum_{i}^{\prime} \frac{3}{2 G_{i}^{2} a^{2}} \exp \left(\frac{G_{i}^{2} a^{2}}{4 A^{2}}\right)-\frac{3}{8 A^{2}}-\frac{A}{\sqrt{\pi}},
$$

where $A$ is an arbitrary number to be chosen to speed up the convergency of the sum. In Eq. (7) we set $A=\sqrt{3} a /\left(2 r_{T}\right)$. Eq. (7) has simple meaning. The function $g_{1}(\boldsymbol{r})$ describes uncorrelated vibrations of ions near their lattice sites, with a symmetric Gaussian probability distribution characterized by the rms displacement $r_{T}$. Thus, $U_{1}$ is the energy of a point-like ion in a lattice site $\left(\zeta Z^{2} e^{2} / a\right)$ with two corrections. The first correction, which contains $r_{T}^{2}$, is equal to the energy required to shift an ion at a distance $r_{T}$ from the center of a sphere filled by a rigid electron background. The second correction refers to electrostatic interaction of two ions at a mean distance $R_{i}$. Specifically, it is the difference of the exact interaction energy for Gaussian ion density distributions and the electrostatic energy of point-like ions; this difference is exponentially small.

Let us take the second term in Eq. (6) and change the order of summation and integration. The integration of $g_{1}(\boldsymbol{r})$ gives $Z^{2} e^{2} /\left(2 R_{i}\right)\left[1-\operatorname{erfc}\left(\sqrt{3} R_{i} / 2 r_{T}\right)\right]$. The term containing erfc exactly cancels similar term in $U_{1}$. Then

$$
\frac{U}{N T \Gamma}=\zeta+\frac{r_{T}^{2}}{2 a^{2}}+\sum_{i}^{\prime}\left[\frac{\sqrt{N\left(\boldsymbol{R}_{i}\right)}}{16 \pi^{3 / 2}} \int \frac{a}{r} \mathrm{e}^{-N_{\alpha \beta}\left(\boldsymbol{R}_{i}\right) x_{i \alpha} x_{i \beta} / 4} \mathrm{~d} \boldsymbol{r}-\frac{a}{2 R_{i}}\right],
$$

where $\Gamma=Z^{2} e^{2} / a T$ is the Coulomb coupling parameter. To analyze this expression, let us take any term under the sum over $i$ and introduce a local coordinate frame with $z$ axis along $\boldsymbol{R}_{i}$. The main contribution into the integral comes from a small vicinity of $\boldsymbol{r}$ near $\boldsymbol{R}_{i}$. Thus, $1 / r$ can be expanded in series of $\boldsymbol{x}_{i}$. The leading expansion term, $1 / R_{i}$, after the integration, will be canceled with $a / 2 R_{i}$. Integrals containing odd products of $\boldsymbol{x}_{i}$ components vanish, while integrals containing even products of $\boldsymbol{x}_{i}$ components are taken by introducing a coordinate frame which diagonalizes $N_{\alpha \beta}=$ $N_{\alpha \beta}\left(\boldsymbol{R}_{i}\right)$. After performing integration in that frame we can rotate the coordinate axis and return to the initial frame. For instance, consider the integral which contains a 
product of four $\boldsymbol{x} \equiv \boldsymbol{x}_{i}$ components. Let $T_{\alpha i}$ be the rotation matrix. Then

$$
\begin{aligned}
\int x_{\mu} x_{v} x_{\lambda} x_{\xi} \mathrm{e}^{-N_{\alpha \beta} x_{\alpha} x_{\beta} / 4} \mathrm{~d} \boldsymbol{x} & =T_{\mu i} T_{v k} T_{\lambda l} T_{\xi m} \int x_{i}^{\prime} x_{k}^{\prime} x_{l}^{\prime} x_{m}^{\prime} \mathrm{e}^{-\sum_{j} N_{j j}^{\prime} x_{j}^{\prime 2} / 4} \mathrm{~d} \boldsymbol{x}^{\prime} \\
& =4 T_{\mu i} T_{v k} T_{\lambda l} T_{\xi m}\left(V_{i k}^{\prime} V_{l m}^{\prime}+V_{i l}^{\prime} V_{k m}^{\prime}+V_{i m}^{\prime} V_{k l}^{\prime}\right) \\
& =4\left(V_{\mu v} V_{\lambda \xi}+V_{\mu \lambda} V_{v \xi}+V_{\mu \xi} V_{\lambda v}\right) .
\end{aligned}
$$

The primed quantities refer to a coordinate frame, where $N_{\alpha \beta}$ is diagonal.

In this way after some algebra we finally obtain

$$
\frac{U}{N T}=\zeta \Gamma+A_{0}^{\mathrm{HL}}+\frac{A_{1}^{\mathrm{HL}}}{\Gamma}+\frac{A_{2}^{\mathrm{HL}}}{\Gamma^{2}}+\cdots,
$$

where $A_{0}^{\mathrm{HL}}$ is the harmonic coefficient given by

$$
A_{0}^{\mathrm{HL}}=\Gamma \frac{r_{T}^{2}}{2 a^{2}}+\sum_{i}^{\prime} \frac{a \Gamma}{2 R_{i}^{3}}\left(v_{x x}+v_{y y}-2 v_{z z}\right),
$$

while the first two anharmonic coefficients are

$$
\begin{aligned}
A_{1}^{\mathrm{HL}}= & \sum_{i} \frac{3 a \Gamma^{2}}{R_{i}^{5}}\left\{\frac{3}{4}\left[\left(v_{x x}\right)^{2}+\left(v_{y y}\right)^{2}\right]+2 v_{z z}\left(v_{z z}-v_{x x}-v_{y y}\right)\right. \\
& \left.+\left(v_{x y}\right)^{2}+\frac{1}{2} v_{x x} v_{y y}-4\left[\left(v_{x z}\right)^{2}+\left(v_{y z}\right)^{2}\right]\right\}, \\
A_{2}^{\mathrm{HL}}= & \sum_{i} \frac{30 a \Gamma^{3}}{R_{i}^{7}}\left\{\frac{5}{8}\left(v_{x x}\right)^{3}+\frac{5}{8}\left(v_{y y}\right)^{3}+\frac{3}{2}\left(v_{x y}\right)^{2}\left(v_{x x}+v_{y y}\right)\right. \\
& -9\left[\left(v_{x z}\right)^{2} v_{x x}+\left(v_{y z}\right)^{2} v_{y y}\right]+\frac{3}{8} v_{x x} v_{y y}\left(v_{x x}+v_{y y}\right) \\
& -3\left[\left(v_{x z}\right)^{2} v_{y y}+\left(v_{y z}\right)^{2} v_{x x}+\left(v_{x y}\right)^{2} v_{z z}\right]-12 v_{x y} v_{x z} v_{y z} \\
& -\frac{9}{4} v_{z z}\left[\left(v_{x x}\right)^{2}+\left(v_{y y}\right)^{2}\right]+12 v_{z z}\left[\left(v_{x z}\right)^{2}+\left(v_{y z}\right)^{2}\right] \\
& \left.-\frac{3}{2} v_{x x} v_{y y} v_{z z}+3\left(v_{z z}\right)^{2}\left(v_{x x}+v_{y y}\right)-2\left(v_{z z}\right)^{3}\right\},
\end{aligned}
$$

where $v_{\alpha \beta}=v_{\alpha \beta}\left(\boldsymbol{R}_{i}\right)$. The index "HL" indicates that the coefficients are calculated within the HL model. Eqs. (11)-(14) were obtained in Refs. [5,8] but the derivation presented here is more straightforward.

The first term, $A_{0}^{\mathrm{HL}}$, contains the expression

$$
v_{x x}+v_{y y}-2 v_{z z}=\frac{3 \hbar}{2 M}\left\langle\frac{1-3\left(e_{v z}\right)^{2}}{\omega_{v}} \cos \left(\boldsymbol{q} \cdot \boldsymbol{R}_{i}\right) \operatorname{coth}\left(\frac{\hbar \omega_{v}}{2 T}\right)\right\rangle_{\mathrm{ph}} .
$$


Taking into account Eqs. (12), (15) and (3), $A_{0}^{\mathrm{HL}}$ can be rewritten as

$$
\begin{aligned}
A_{0}^{\mathrm{HL}} & =\frac{3}{4 T} \hbar \omega_{\mathrm{p}}^{2}\left\langle\frac{1}{\omega_{v}}\left(\frac{1}{3}+\frac{1}{4 \pi n} \sum_{\boldsymbol{R}_{i}}^{\prime} \frac{R_{i}^{2}-3\left(\boldsymbol{R}_{i} \cdot \boldsymbol{e}_{v}\right)^{2}}{R_{i}^{5}} \cos \left(\boldsymbol{q} \cdot \boldsymbol{R}_{i}\right)\right) \operatorname{coth}\left(\frac{\hbar \omega_{v}}{2 T}\right)\right\rangle_{\mathrm{ph}} \\
& =\frac{3 \hbar \omega_{\mathrm{p}}^{2}}{4 T}\left\langle\frac{D_{\alpha \beta}(\boldsymbol{q}) e_{v \alpha} e_{v \beta}}{\omega_{v}} \operatorname{coth}\left(\frac{\hbar \omega_{v}}{2 T}\right)\right\rangle_{\mathrm{ph}} \\
& =\frac{3}{4}\left\langle\frac{\hbar \omega_{v}}{T} \operatorname{coth}\left(\frac{\hbar \omega_{v}}{2 T}\right)\right\rangle_{\mathrm{ph}}=\frac{E_{\mathrm{ph}}}{2 N T},
\end{aligned}
$$

where $D_{\alpha \beta}(\boldsymbol{q})$ is the dynamic matrix, $E_{\mathrm{ph}}$ is the total energy of non-interacting phonons (including the kinetic energy of ions). The quantity $E_{\mathrm{ph}}$ is the sum of the kinetic and potential energies of oscillating ions, which are equal due to the virial theorem. It turns out that the HL model gives exact coefficient $A_{0}$ in the expansion (11). For that reason we will omit the index "HL" in $A_{0}$. Eq. (16) is more practical than Eq. (12), because it does not require calculating matrix $v_{\alpha \beta}\left(\boldsymbol{R}_{i}\right)$.

Let us consider the asymptotic behavior of the matrix $\Gamma v_{\alpha \beta}$ at a fixed $\boldsymbol{R}_{i}$. In the high-temperature classical limit $\left(\hbar \omega_{\mathrm{p}} / T \equiv \theta \ll 1\right)$ the asymptote of $\Gamma v_{\alpha \beta}$ is defined by the expansion of $\tanh \left(\hbar \omega_{v} / 2 T\right)$ in powers of $\hbar \omega_{v} / 2 T$. It contains only even powers of $\theta$. However the first quantum correction $\Gamma v_{\alpha \beta}^{\mathrm{q} 1}$ (that should be $\propto \theta^{2}$ ) vanishes after the integration over the Brillouin zone. For demonstrating this, let us calculate a diagonal matrix element $\Gamma v_{\alpha \alpha}^{\mathrm{q} 1}$ in an arbitrary coordinate system

$$
\begin{aligned}
\Gamma v_{\alpha \alpha}^{\mathrm{q} 1} & \propto\left\langle e_{v \alpha} e_{v \alpha} \cos \left(\boldsymbol{q} \cdot \boldsymbol{R}_{i}\right)\right\rangle_{\mathrm{ph}}=\frac{1}{3}\left\langle\left(\sum_{s=1}^{3} e_{v \alpha} e_{v \alpha}\right) \cos \left(\boldsymbol{q} \cdot \boldsymbol{R}_{i}\right)\right\rangle_{\mathrm{ph}} \\
& =\frac{1}{3}\left\langle\cos \left(\boldsymbol{q} \cdot \boldsymbol{R}_{i}\right)\right\rangle_{\mathrm{ph}}=0 .
\end{aligned}
$$

Accordingly, $\Gamma v_{\alpha \beta}^{\mathrm{q} 1} \equiv 0$. In the low-temperature quantum limit $(\theta \gg 1)$ the asymptote of $\Gamma v_{\alpha \beta}$ contains the main term $\propto \theta$ (determined by zero point vibrations) and small thermal corrections. As seen from Eq. (4), the main contribution into the thermal corrections comes from the central part of the Brillouin zone and can be extracted by expanding $\cos \left(\boldsymbol{q} \cdot \boldsymbol{R}_{i}\right)$ in powers of $\left(\boldsymbol{q} \cdot \boldsymbol{R}_{i}\right)$ and using the dispersion equations of acoustic modes. In this case the asymptote contains only odd powers of $\theta$. The first thermal correction $\left(\propto \theta^{-1}\right)$ is

$$
\Gamma v_{\alpha \beta}^{\mathrm{th} 1}=\frac{3 \hbar}{2 M}\left\langle\frac{e_{v \alpha} e_{v \beta}}{\omega_{v}} \frac{1}{\exp \left(\hbar \omega_{v} / T\right)-1}\right\rangle_{\mathrm{ph}}=\frac{1}{3} r_{T 1}^{2} \delta_{\alpha \beta},
$$

where $r_{T 1}^{2}$ is the first thermal correction to the $r_{T}^{2}$. The last equality is valid for fcc and bcc crystals. Thus, the first thermal correction corresponds to highly correlated collective ion motion which does not change the pair distribution function Eq. (2) and the electrostatic energy Eq. (1).

To calculate $A_{1}^{\mathrm{HL}}$ and $A_{2}^{\mathrm{HL}}$ we need to include only a few terms. On the other hand, Eq. (12) for the harmonic term is impractical, and one is forced to use Eq. (16). 


\section{Anharmonic corrections to the electrostatic energy of a Coulomb crystal}

It this section we present the results of the HL calculations of the anharmonic corrections to the electrostatic energy of a Coulomb crystal.

Our main approximation consists in replacing the exact pair correlation function of ions, $g(r)$, by the function obtained in the HL approximation. To demonstrate the accuracy of this approximation, in Fig. 1 we compare exact functions with HL ones for a bec crystal at $\Gamma=200$ and two values of the quantum parameter, $\theta=0$ and 24.5. In the classical crystal $(\theta=0)$, the exact function $g(r)$ was calculated by Stringfellow et al. [10] using the classical Monte Carlo (MC) scheme. In the quantum crystal $(\theta=24.5)$ the exact function was calculated by Pollock and Militzer [11] within the path integral Monte Carlo (PIMC) method. Both exact curves are presented with kind permission of the authors. The agreement of the exact and HL curves is seen to be quite satisfactory. This agreement is expected to become worse at lower $r$ (not shown in Fig. 1) where the HL approximation is inaccurate (e.g., Ref. [6]). One the other hand, at large $r$ the HL results are thought to be highly accurate. In this limit the HL method becomes most efficient for calculating $g(r)$. The first anharmonic correction to the Coulomb energy, calculated with the HL $g(r)$ in the classical limit, differs from the exact anharmonic correction only by $2 \%$ (Ref. [5] and a discussion below). In the quantum limit $(\theta=24.5)$ the relative difference between exact and HL $g(r)$ remains nearly the same as in the classical limit. This allows us to expect that our model gives sufficiently accurate values of the first anharmonic correction for any $\theta$.

Fig. 2 shows three expansion coefficients $A_{i}^{\mathrm{HL}}$ (for $i=0,1$, and 2, Eqs. (12)-(14)) as functions of $\theta$ for fcc and bcc lattices. Notice the asymptotic values $A_{1}^{\mathrm{HL}} \approx 10.64$ and 5.61 for bcc and fcc lattices, respectively, in the classical limit $(\theta \ll 1)$, and the behavior $A_{1}^{\mathrm{HL}} \propto \theta^{2}$ in the quantum limit $(\theta \gg 1)$.

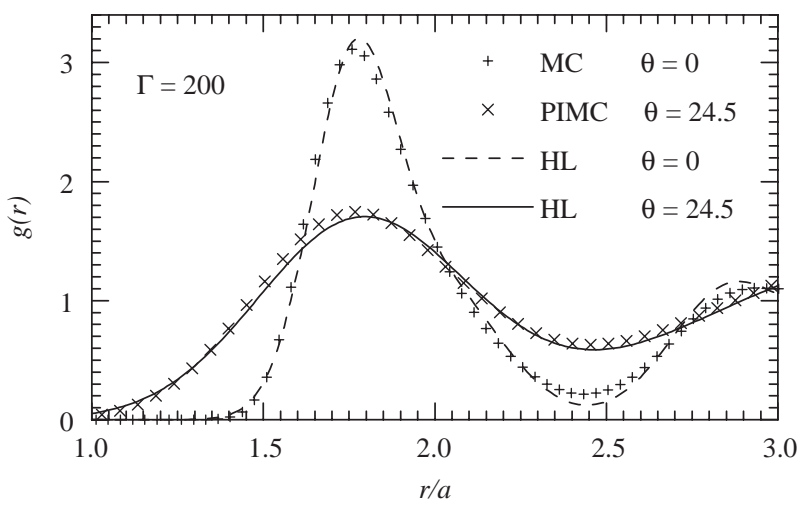

Fig. 1. Pair correlation function of a Coulomb crystal for $\Gamma=200$ and two values $\theta=0$ and 24.5. Crosses show the results of classical Monte Carlo [10] and path integral Monte Carlo [11] calculations. Lines are HL calculations. 


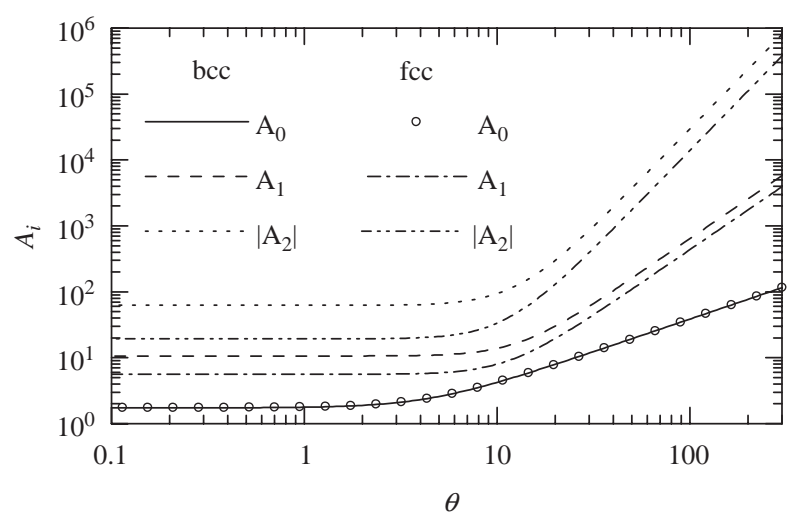

Fig. 2. First three expansion coefficients, $A_{0}, A_{1}^{\mathrm{HL}}$, and $A_{2}^{\mathrm{HL}}=-\left|A_{2}^{\mathrm{HL}}\right|$, for bcc and fcc lattices versus $\theta$.

In the classical limit the exact values of the anharmonic correction found by Dubin [4] are: $A_{1}=10.85$ for bcc and $A_{1}=12.34$ for fcc lattice. In the formalism of Ref. [4], $A_{1}$ can be written as a difference of two terms, which we call here as $A_{11}$ and $A_{12}$ : $A_{1}=A_{11}-A_{12}$. Dubin [4] found $A_{12}=10.69$ for bcc and $A_{12}=5.63$ for fcc lattice. As shown in Ref. [5], $A_{1}^{\mathrm{HL}}=+A_{12}$ and $A_{1}^{\mathrm{HL}}=10.64$ for bcc. Our result is $A_{1}^{\mathrm{HL}}=$ 10.642 for bcc and $A_{1}^{\mathrm{HL}}=5.611$ for fcc lattice in the classical limit. These numbers differ from the results of Ref. [4] by $0.45 \%$ for bcc and by $0.34 \%$ for fcc lattice. Notice also that in the case of bcc lattice $A_{1}^{\mathrm{HL}}$ differs from $A_{1}$ only by $2 \%$. Hence, the HL model gives surprisingly accurate values of $A_{0}$ and $A_{1}$ for the bcc lattice in the classical limit.

As for the coefficient $A_{2}^{\mathrm{HL}}$ (Fig. 2), it behaves as $A_{2}^{\mathrm{HL}} \approx-62.4$ and -19.4 in the classical bcc and fcc lattices, respectively, and as $A_{2}^{\mathrm{HL}} \propto \theta^{3}$ in the quantum lattice. Our approximation, which neglects the anharmonicity of the pair distribution function, is too crude for an accurate determination of $A_{2}$. As a result, our numerical value of $A_{2}^{\mathrm{HL}}$ in the classical limit for the bcc lattice disagrees (even has the wrong sign) with the value $A_{2} \approx 247$, deduced from MC data (Table V of Ref. [12]). Our aim here is to point out the quantum asymptote $A_{2}^{\mathrm{HL}} \propto \theta^{3}$ and, possibly, the analytic expression (14), rather than numerical values of $A_{2}^{\mathrm{HL}}$.

In contrast to the coefficients $A_{1}^{\mathrm{HL}}$ and $A_{2}^{\mathrm{HL}}$, the main coefficient $A_{0}$ is almost independent of the lattice type (bcc or fcc); $A_{0}$ has been calculated previously (Ref. [13]) and is shown here for completeness.

The asymptotic behavior of $A_{i}^{\mathrm{HL}}$ in the classical limit is determined by the asymptotes of the matrix $\Gamma v_{\alpha \beta}\left(\boldsymbol{R}_{i}\right)$. As shown earlier, only even powers of $\theta\left(\theta^{0}, \theta^{4}\right.$, $\theta^{6}, \ldots$; excluding $\theta^{2}$ ) appear in the series expansion of this matrix in the classical limit. Accordingly, the expansions of $A_{1}^{\mathrm{HL}}$ and $A_{2}^{\mathrm{HL}}$ behave as $c_{1}+c_{2} \theta^{4}+c_{3} \theta^{6}+\ldots$ In contrast, $A_{0}$ contains mean-squared ion displacement $r_{T}^{2}$, and the associated $\theta^{2}$ expansion term, $A_{0}=c_{1}+c_{2} \theta^{2}+c_{3} \theta^{4}+\ldots$.

The asymptotic behavior of $A_{i}^{\mathrm{HL}}$ in the quantum limit is more complicated. The main contribution into $A_{i}^{\mathrm{HL}}$ comes from zero point vibrations. As discussed above, 
Table 1

Fit parameters for Eq. (19)

\begin{tabular}{|c|c|c|c|c|c|c|}
\hline & \multicolumn{3}{|l|}{ bcc } & \multicolumn{3}{|l|}{ fcc } \\
\hline & $A_{0}$ & $A_{1}^{\mathrm{HL}}$ & $A_{2}^{\mathrm{HL}}$ & $A_{0}$ & $A_{1}^{\mathrm{HL}}$ & $A_{2}^{\mathrm{HL}}$ \\
\hline$C_{1}$ & 1.5 & 10.6424 & -62.4052 & 1.5 & 5.611 & -19.389 \\
\hline$C_{2}$ & 9.303 & 2.2705 & 2.2783 & 9.41506 & 2.5196 & 2.5375 \\
\hline$C_{3}$ & 25.242 & 1.3948 & 1.4224 & 26.1463 & 1.9998 & 2.0555 \\
\hline$C_{4}$ & 15.196 & 0.31981 & 0.322917 & 16.9613 & 0.58583 & 0.6091 \\
\hline$C_{5}$ & 3.58 & 0.01 & 0.01338 & 4.731 & 0.00589 & 0.00094 \\
\hline$C_{6}$ & 3.7482 & 2.2705 & 2.2783 & 3.86055 & 2.5196 & 2.5375 \\
\hline$C_{7}$ & 2.3244 & 0.5318 & 0.53081 & 2.57606 & 0.75471 & 0.758 \\
\hline$\delta$ & $3 \times 10^{-5}$ & $8 \times 10^{-5}$ & $7 \times 10^{-5}$ & $7 \times 10^{-6}$ & $4 \times 10^{-5}$ & $1.3 \times 10^{-4}$ \\
\hline
\end{tabular}

the first-order thermal correction to the matrix $\Gamma v_{\alpha \beta}\left(\boldsymbol{R}_{i}\right)$ does not change the electrostatic energy. The higher order expansion terms cannot be obtained directly from the expansion of the matrix $\Gamma v_{\alpha \beta}\left(\boldsymbol{R}_{i}\right)$ in powers of $\theta$, because this expansion does not converge uniformly with respect to $\boldsymbol{R}_{i}$. However, the same situation takes place also for the harmonic coefficient $A_{0}$, whose exact quantum asymptote coincides with the asymptote obtained using a formal expansion of $\Gamma v_{\alpha \beta}\left(\boldsymbol{R}_{i}\right)$ in powers of $\theta$. Based on this and for the sake of fitting the numerical results we assume that the asymptotes of other coefficients $A_{i}^{\mathrm{HL}}$ can be written as $A_{i}^{\mathrm{HL}}=c_{1} \theta^{i+1}+c_{2} \theta^{i-3}+$ $c_{3} \theta^{i-5}+\ldots$ Accordingly, we can propose the fit expression

$$
A_{i}^{\mathrm{HL}}=C_{1}\left(\frac{1+C_{2} t^{2}+C_{3} t^{4}+C_{4} t^{6}+C_{5} t^{8}}{1+C_{6} t^{2}+C_{7} t^{4}+C_{8} t^{6}}\right)^{(i+1) / 2} \text { for } i=0,1,2,
$$

where $t=0.1 \theta$, and $C_{8}=C_{5} C_{7} / C_{4}$ (to reach agreement with quantum series expansions). To satisfy the asymptotes in the classical regime we can also impose the condition $C_{6}=C_{2}$ for $A_{1}$ and $A_{2}$. Because $A_{0}=1.5$ in the classical limit, we can set $C_{1}=1.5$. We have fitted our numerical results for bcc and fcc lattices with $\theta$ ranging from 0.1 to 300 . The fit parameters $C_{1}-C_{7}$ and the maximum fit error $\delta$ are summarized in Table 1. An rms relative error over a representative grid of data points, including the points from the classical and quantum domains, is typically twice lower than the maximum error for all $A_{i}^{\mathrm{HL}}$ and both crystal types. The fits are seen to be highly accurate.

The temperature dependence of the thermal part of anharmonic corrections to the electrostatic energy and the thermal energy of the harmonic lattice is the same $\left(U_{\text {th }} \propto T^{4}\right)$ in the quantum limit.

\section{Anharmonicity of crystals in white dwarf cores and neutron star envelopes}

For illustration, let us analyze anharmonicity of Coulomb crystals in the cores of white dwarfs and the envelopes of neutron stars. 
Let us define the energy of zero-point ion vibrations per one ion

$$
E_{0}=\lim _{T \rightarrow 0} E(T)-E_{\mathrm{M}},
$$

where $E_{\mathrm{M}}=\zeta Z^{2} e^{2} / a$ is the Madelung energy and $E(T)$ is the crystal energy (per ion) at a temperature $T$. Let us introduce also the thermal energy of the crystal $E_{\mathrm{th}}(T) \equiv$ $E(T)-E_{0}-E_{\mathrm{M}}$. In each energy, $E_{0}$ and $E_{\mathrm{th}}$, we select a harmonic component (that corresponds to the purely harmonic Hamiltonian): $E_{0}^{\mathrm{HL}}=3 u_{1} \hbar \omega_{\mathrm{p}} / 2$ $\left(u_{1} \equiv\left\langle\omega_{v} / \omega_{\mathrm{p}}\right\rangle_{\mathrm{ph}} \approx 0.5113875\right.$ and 0.5131940 , for bcc and fcc crystals, respectively) and $E_{\mathrm{th}}^{\mathrm{HL}}$. These harmonic components have been studied in Ref. [13]. Exact anharmonic corrections to these components will be denoted by $\delta E_{0} \equiv E_{0}-E_{0}^{\mathrm{HL}}$ and $\delta E_{\mathrm{th}} \equiv E_{\mathrm{th}}-E_{\mathrm{th}}^{\mathrm{HL}}$. Approximate anharmonic corrections, calculated in the frame of our approximation by taking into account the first anharmonic correction $A_{1}^{\mathrm{HL}}$, will be denoted as $\delta E_{0}^{(1)}$ and $\delta E_{\mathrm{th}}^{(1)}$.

For example, consider the ${ }^{12} \mathrm{C}$ matter of the density $\rho=10^{6} \mathrm{~g} \mathrm{~cm}^{-3}$, characteristic of cores of white dwarfs and envelopes of neutron stars. In this case the melting temperature is $T_{\mathrm{m}} \approx 2.044 \times 10^{6} \mathrm{~K}$, and the plasma temperature $T_{\mathrm{p}} \equiv \hbar \omega_{\mathrm{p}} \approx$ $3.916 \times 10^{6} \mathrm{~K}$. The Madelung energy (per ion in temperature units) appears to be $E_{\mathrm{M}} \approx-3.205 \times 10^{8} \mathrm{~K}$; the harmonic energy of zero-point ion vibrations is $E_{0}^{\mathrm{HL}} \approx$ $3 \times 10^{6} \mathrm{~K}$, and its anharmonic correction is $\delta E_{0}^{(1)} \sim 1.5 \times 10^{4} \mathrm{~K}$. These values are almost the same for bcc and fcc lattices. The zero-point vibration energy is a small correction to the Madelung energy. The anharmonic correction $\delta E_{0}^{(1)}$ can be neglected in comparison with the Madelung energy.

Fig. 3 shows the thermal energy per ion $E_{\text {th }}^{\mathrm{HL}}$ and its first anharmonic correction $\delta E_{\mathrm{th}}^{(1)}$ as a function of temperature for a ${ }^{12} \mathrm{C}$ bcc crystal at $\rho=10^{6} \mathrm{~g} \mathrm{~cm}^{-3}$. We also display the electron thermal energy $E_{\mathrm{e}}^{\text {th }}(T)=E_{\mathrm{e}}(T)-E_{\mathrm{e}}(0)$. The upper horizontal

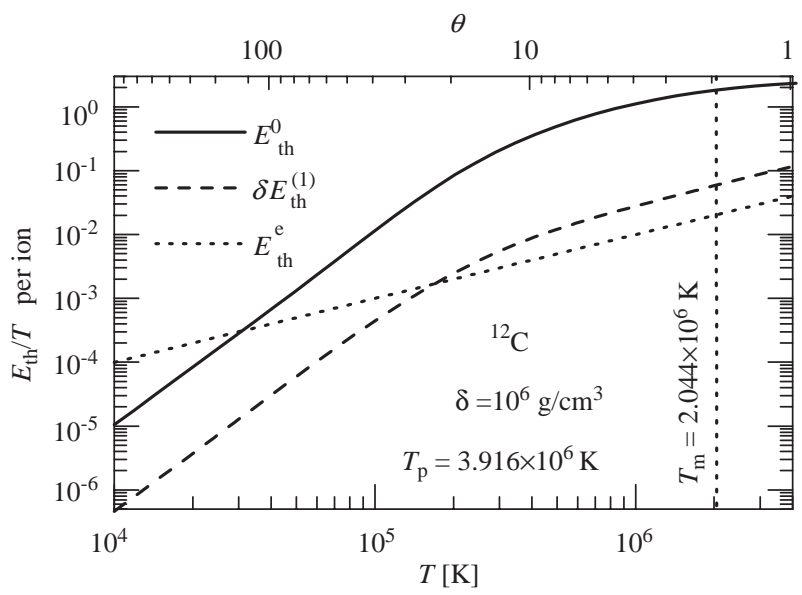

Fig. 3. Thermal energy (per ion) $E_{\mathrm{th}}^{\mathrm{HL}}$ and its first anharmonic correction $\delta E_{\mathrm{th}}^{(1)}$ as a function of temperature for crystalline (bcc) ${ }^{12} \mathrm{C}$ matter of the density $\rho=10^{6} \mathrm{~g} \mathrm{~cm}^{-3}$. We also show the electron thermal energy $E_{\mathrm{e}}^{\text {th }}$ (per ion). The upper horizontal scale gives the values of $\theta$. The vertical dotted line shows the melting temperature $T_{\mathrm{m}}$. 
scale gives the values of $\theta$. The vertical dotted line indicates the melting temperature $T_{\mathrm{m}}$. The curves in the crystalline phase are extended continuously beyond $T_{\mathrm{m}}$ just for illustration. At low temperatures $T \ll T_{\mathrm{p}}$ the energy $\delta E_{\mathrm{th}}^{(1)}$ constitutes $4 \%$ of the total harmonic thermal energy. With growing temperature the ratio $\delta E_{\text {th }}^{(1)} / E_{\text {th }}^{\mathrm{HL}}$ decreases to the minimum value $\sim 2.4 \%$ at $T \sim 7 \times 10^{5} \mathrm{~K}$. With further growth of $T$ the anharmonicity of the crystal increases because of the increase of the amplitude of ion vibrations. At low temperatures $\left(T \ll T_{\mathrm{p}}\right)$ only a small fraction of phonons with low frequencies contributes to the thermal energy of the matter. As a result, at $T \lesssim 3 \times 10^{4} \mathrm{~K}$ the main contribution into the thermal energy comes from degenerate electrons.

Let us notice that the ratio $\delta E_{\mathrm{th}}^{(1)} / E_{\mathrm{th}}^{\mathrm{HL}}$ behaves as $\propto \rho^{1 / 6}$ at $T \ll T_{\mathrm{p}}$ and as $\propto \rho^{-1 / 3}$ at $T \gg T_{\mathrm{p}}$.

Notice also that in the classical limit near the melting point the total anharmonic correction, known from the Monte Carlo simulations (e.g., Ref. [14]), exceeds $\delta E_{\text {th }}^{(1)}$ by about $80 \%$.

We expect that the numerical values presented above are valid at least for orderof-magnitude estimates. They indicate that Coulomb crystals in white dwarf cores and neutron star envelopes are sufficiently harmonic. More accurate consideration of anharmonic corrections is required for incorporating these corrections into computer codes which simulate, for instance, thermal evolution of white dwarfs. However, our calculations give accurate values of approximate quantities which, in fact, represent certain terms of exact anharmonic contribution, useful for deriving exact results in the future.

\section{Conclusions}

We have used the harmonic-lattice approximation to calculate the first- and second-order anharmonic corrections to the electrostatic energy of a Coulomb crystal with bcc and fcc lattices. Our calculations cover the high-temperature classical regime, the low-temperature quantum regime, and the intermediate regime. In the classical regime, the first-order anharmonic term for bcc lattice is shown to be in a good agreement with the previous results (e.g., Refs. [4,5]). In our model the thermal part of the anharmonic corrections to the electrostatic energy in the quantum limit have the same temperature dependence as thermal energy of the harmonic lattice $\left(E_{\mathrm{th}} \propto T^{4}\right)$. The harmonic energy and the first- and secondorder anharmonic coefficients are fitted by simple analytical expressions with the maximum error of $\lesssim 10^{-4}$. For the typical conditions in envelopes of neutron stars and cores of white dwarfs the anharmonic corrections do not exceed a few percent of the harmonic energy.

\section{Acknowledgements}

We are grateful to D.G. Yakovlev and H.E. De Witt for discussions, and to R. Pollock for providing us with the data on PIMC $g(r)$. The work was partly 
supported by the RFBR Grant 02-02-17668, the grant of the Dynasty Foundation, RFBR-MAC Grant 03-02-06803, and RLSSF Grant 1115.2003.2.

\section{References}

[1] S.Ya. Rakhmanov, Zh. Eksper. Teor. Fiz. 75 (1978) 160.

[2] W.M. Itano, J.J. Bollinger, J.N. Tan, B. Jelencović, X.-P. Xuang, D.J. Wineland, Science 279 (1998) 686.

[3] A.A. Maraduddin, E.W. Montroll, G.H. Weiss, Theory of Lattice Dynamics in the Harmonic Approximation, Academic Press, New York, 1963.

[4] D.H.E. Dubin, Phys. Rev. A 42 (1990) 4972.

[5] D.A. Baiko, D.G. Yakovlev, H.E. DeWitt, W.L. Slattery, Phys. Rev. E 61 (2000) 1912.

[6] A.I. Chugunov, D.A. Baiko, D.G. Yakovlev, H.E. DeWitt, W.L. Slattery, Physica A 323 (2003) 413.

[7] R.C. Albers, J.E. Gubernatis, preprint of the LASL LA-8674-MS, 1981.

[8] D.A. Baiko, Ph.D. Thesis, Ioffe Physical-Technical Institute, St.-Petersburg, Russia, 2000.

[9] M. Born, K. Huang, Dynamical Theory of Crystal Lattices, Claredon Press, Oxford, 1954.

[10] G.S. Stringfellow, H.E. DeWitt, W.L. Slattery, Phys. Rev. A 41 (1990) 1105.

[11] E.L. Pollock, B. Militzer, Phys. Rev. Lett. 92 (2004) 021101.

[12] R.T. Farouki, S. Hamaguchi, Phys. Rev. E 47 (1993) 4330.

[13] D.A. Baiko, A.Y. Potekhin, D.G. Yakovlev, Phys. Rev. E 64 (2001) 057402.

[14] H. DeWitt, W. Slattery, D. Baiko, D. Yakovlev, Contrib. Plasma Phys. 41 (2001) 251. 